\title{
Rezidivtherapie aggressiver Lymphome
}

Hintergrund und Fragestellung: Standardtherapie für Rezidive aggressiver Lymphome ist DHAP (Dexamethason, Cytarabin, Cisplatin), bei B-Zelllymphomen mit Rituximab ergänzt und begleitet von supportiven Maßnahmen. Dennoch kommt es bei den bereits vorbehandelten Patienten zu schwergradigen Neutro- und Thrombopenien, cisplatinbedingter Tubulusschädigung mit konsekutiver Niereninsuffizenz und selten zu akuter Neurotoxizität (Cytarabin). Nach zwei bis drei Zyklen ist regelhaft eine autologe Stammzelltransplantation möglich. Das Therapieansprechen ist prognosebestimmend. Ziel ist deshalb eine möglichst tiefe Remission. Weniger toxische Alternativen sind wünschenswert.

Patienten und Methoden: In der NCIC-LY-12-Studie wurde untersucht, inwieweit die Patienten von einem Austausch von Cytarabin gegen Gemcitabin profitieren. Behandelt wurden insgesamt 619 Patienten mit rezidivierten aggressiven Lympho-

Originalie

Crump M et al. Randomized comparison of gemcitabine, dexamethasone, and cisplatin versus dexamethasone, cytarabine, and cisplatin chemotherapy before autologous stem-cell transplantation for relapsed and refractory aggressive lymphomas: NCIC-CTG LY-12. J Clin Oncol. 2014;32(31):3490-6 me (90\% B-Zelllymphome). $30 \%$ der Patienten waren primär refraktär, und $40 \%$ hatten ein Frührezidiv $(<1 \mathrm{Jahr}$ nach Primärtherapie). Damit stellte die Studienpopulation eine Gruppe mit sehr schlechter Prognose dar. Die Patienten erhielten 1:1 randomisiert entweder DHAP oder GDP (Gemcitabin, Dexamethason,
Cisplatin). Bei Ansprechen wurde autolog transplantiert. Dies gelang bei 307 Patienten. Primärer Endpunkt war die Ansprechrate nach zwei Zyklen, sekundäre Endpunkte waren ereignisfreies und Gesamtüberleben, sowie Stammzellmobilisierbarkeit und Lebensqualität.

Beide Schemata waren vergleichbar wirksam bezüglich Ansprechrate (DHAP: 44 vs. GDP: $45 \%$; $\mathrm{p}=0,84$ ), ereignisfreiem 4-Jahres-Überleben (26 vs. $26 \%$; Hazard Ratio [HR] 0,99; p = 0,95 ) und Gesamtüberleben ( 39 vs. $39 \%$; HR 1,03; $p=0,78$ ). Dies ist bemerkenswert, auch vor dem Hintergrund der reduzierten Cisplatin-Dosis des GDP-Regimes $\left(75 \mathrm{mg} / \mathrm{m}^{2}\right.$ statt $100 \mathrm{mg} / \mathrm{m}^{2}$ im DHAP-Schema). Für die Patienten, die transplantiert werden konnten, zeigte sich ein ereignisfreies Überleben von $45 \%$ und ein Gesamtüberleben von $68 \%$. Allerdings spachen die primär refraktären Patienten nur zu $35 \%$ an und die mit Frührezidiven nur zu $40 \%$. Patienten mit Rezidiv > 1 Jahr nach Primärtherapie hatten eine deutlich günstigere Prognose.

Auch die Stammzellmobilisierbarkeit war vergleichbar im GDP- und DHAP-Arm (88 vs. $82 \%$; $\mathrm{p}=0,14$ ), es gab 6 Mobilisierungsversager im GDP-Arm und 7 im DHAP-Arm. Die Rate an febrilen Neutropenien war im GDP-Arm geringer (9 vs. $23 \%$ ), ebenso der Anteil der Patienten, die Plättchentransfusionen (31 vs. $47 \%$ ) oder Hospitalisationen ( 37 vs. $99 \%$ ) benötigten.

Schlussfolgerung der Autoren: GDP ist so effektiv wie DHAP bei geringerer Nebenwirkungsrate und besser Lebensqualität.

Schlüsselwörter: DHAP - Gemcitabin - aggresive Lymphome

\section{- Kommentar von Jörg T. Bittenbring, Homburg/Saar}

\section{„GDP kann als Basis für weitere Therapieverbesserungen dienen“}

Michael Crump und Kollegen zeigen in dieser für Rezidivpatienten ungewöhnlich großen Studie, dass GDP bei Patienten mit rezidivierten aggressiven Lymphomen eine äquipotente und weniger toxische Kombination ist als DHAP. Eine ambulante Durchführung der Chemotherapie war in 50\% der Fälle möglich gegenüber nur $1 \%$ bei DHAP. Die Stammzellmobilisierung und Transplantationsrate wurde nicht beeinträchtigt. Das ereignisfreie und Gesamtüberleben konnte für die Hochrisikogruppe „Frührezidiv oder primär refraktär" leider nicht explizit ausgewiesen werden, die Ansprechrate lag jedoch deutlich niedriger als bei Spätrezidiven. In der CORAL-Studie hatten Patienten mit Frührezidiv ein Gesamtüberleben < 20\% [1]. Es ist wichtig, diese Gruppe genauer anzusehen, denn nach den Ergebnissen der DSHNHL-R3-Studie ist mit einer allogenen Transplantation für diese Gruppe vermutlich ein besseres Überleben zu erzielen als mit autologer Transplantation [2].

Auf eine Unterscheidung der DLBCL in aktivierten B-Zelltyp $(A B C)$ oder Keimzentrumstyp (GCB), wie in anderen Rezidivstudien verwendet, wurde verzichtet, so dass Schlussfolgerungen über ein differentielles Ansprechen nicht möglich sind.
GDP ist eine Alternative zu DHAP und kann als Basis für weitere Verbesserungen der Therapie aggressiver Lymphome dienen. Es bietet sich an, zur Reduktion der Nephrotoxizität von GDP Cisplatin durch Oxaliplatin $\left(100 \mathrm{mg} / \mathrm{m}^{2}\right)$ zu ersetzen, wie dies für DHAP bereits in mehreren Phase-II-Studien gezeigt wurde.
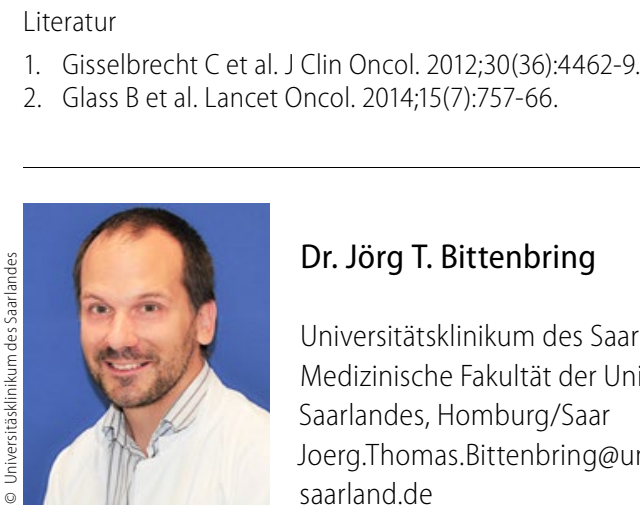

Dr. Jörg T. Bittenbring

Universitätsklinikum des Saarlandes und Medizinische Fakultät der Universität des Saarlandes, Homburg/Saar Joerg.Thomas.Bittenbring@uniklinikumsaarland.de 